\title{
A New Approach to Classical Electrodynamics: Covariant Second-Order Field Variations
}

\author{
William B. Maier II \\ Department of Physics, Naval Postgraduate School (ret.), USA \\ *Corresponding Author: wratv6@gmail.com
}

Copyright $\bigcirc 2018$ by authors, all rights reserved. Authors agree that this article remains permanently open access under the terms of the Creative Commons Attribution License 4.0 International License

\begin{abstract}
This paper describes a formulation of classical electrodynamics without point charges and masses. Maxwell's equations are assumed to be valid at all scales, and the Lorentz force is replaced as a fundamental law of nature by equations derived by a second order variation on a covariant "action" $I=-i \int \mu c d \Omega+\frac{i}{4 \pi c} \int F_{n k}^{n} A_{k} d \Omega+\frac{i}{16 \pi c} \int F_{j k}^{2} d \Omega$ in which the usual term involving mass, $-\sum m c^{2} \sqrt{1-\beta^{2}}$, has been replaced by a covariant mass density $\mu=\frac{1}{c^{4}} T_{i k} u_{i} u_{k}$ associated with the fields. $\mathrm{F}_{\mathrm{ij}}$ and $\mathrm{T}_{\mathrm{ik}}$ are the usual field and energy-momentum tensors, and $u_{i}$ is the four-velocity associated with charge. Distributions having charge of a single sign are found to be stabilized by charge motion in the distribution's self-field, and they have finite extent, charge, and mass. The motion of these charged bodies in an imposed electromagnetic field is shown to obey the Lorentz force law, where the mass is equivalent to the total energy of the electromagnetic field integrated over all space. Ordinary classical electromagnetism is thus reproduced, and in addition, the classical structures of fundamental charged bodies are obtained. The singularities usual to dualistic theories disappear. Classical structures of the electron and proton are briefly discussed.
\end{abstract}

Keywords Electrodynamics, Classical Field Theory, Electromagnetism, Electron Structure, Particle Structure, Second-Order Field Variations, Self-field-stabilized Charged Bodies

\section{Introduction}

Classical electromagnetism is a dualistic theory in which Maxwell's equations give the fields from the charges and their motions, and electrodynamics, i.e., the motions of the charges in the fields, is governed by the Lorentz force plus the radiation reaction. The charges have been taken as separate entities from the fields that cause their motion [1-4]. Charges have been taken to be singular points having infinite charge density, and attempts to identify the field energy with mass have yielded infinite masses.

The purpose of the present work is to formulate a new classical electrodynamics in which the charges arise naturally from the fields in such a way that the singularities of classical electromagnetism disappear. The usual Lorentz force law for the motion of charged bodies emerges from the theory as a derived law, and new electrodynamics equations describing static physical structures for fundamental entities, such as the electron and proton, are obtained.

We formulate a theory of electromagnetism without $\underline{\mathbf{a}}$ priori reference to the structure of the charged bodies involved [3]. Charged entities and their properties must arise from the behavior of the fields.

The basic assumptions are:

1. Fields are everywhere continuous and differentiable, i.e., there are no point, line or surface charges.

2. Special relativity holds.

3. Maxwell's equations hold at all scale dimensions.

4. A covariant energy density $E$ is equivalent to a covariant mass density $\mu=\mathrm{E} / \mathrm{c}^{2}$, where $\mathrm{c}$ is the speed of light in vacuum.

5. The current density $\mathbf{J}=\rho \mathbf{v}$ can be written in terms of the charge density $\rho$ and velocity $\mathbf{v}$, and a given volume element contains charge of only one sign.

6. All interactions are assumed to be purely electromagnetic. All physically observable properties, such as charge, mass, dipole moments, and angular momentum, are obtained from the electromagnetic fields.

7. Stable charged bodies will be surrounded by an equipotential surface.

\section{Preliminaries}

We use $(\mathrm{x}, \mathrm{y}, \mathrm{z}, \mathrm{ict})=\left(\mathrm{x}_{1}, \mathrm{x}_{2}, \mathrm{x}_{3}, \mathrm{x}_{4}\right) . \quad \mathrm{i}=(-1)^{1 / 2}$.

Partial derivatives are designated by $\frac{\partial F}{\partial x_{j}} \equiv F^{j}$ and the 
sum of like quantities by $a_{m}^{2}=a_{m} a_{m}$.

Gaussian units are used throughout.

The transformation formulas [5] for the electromagnetic fields $\mathbf{E}$ and $\mathbf{H}$

$$
\begin{aligned}
\mathbf{E}^{\prime} & =\frac{\vec{\beta} \cdot \mathbf{E}}{\beta^{2}}(1-\gamma) \vec{\beta}+\gamma(\mathbf{E}+\vec{\beta} \times \mathbf{H}) \\
\mathbf{H}^{\prime} & =\frac{\vec{\beta} \cdot \mathbf{H}}{\beta^{2}}(1-\gamma) \vec{\beta}+\gamma(\mathbf{H}-\vec{\beta} \times \mathbf{E})
\end{aligned}
$$

with $\gamma=\frac{1}{\sqrt{1-\beta^{2}}}$ and $\vec{\beta}=\mathbf{v} / c$ are used to derive covariant mass $\mu$ and momentum densities $\zeta_{l}$

$$
\mu=\frac{\varepsilon}{c^{2}}=\frac{1}{c^{4}} T_{i k} u_{i} u_{k}
$$

$$
\zeta_{l}=\frac{1}{4 \pi c^{4}}\left[F_{n d} u_{n}\left(F_{l k} u_{j}-F_{j k} u_{l}\right) u_{j}\right]=-\left(\mu u_{l}+\frac{1}{c^{2}} T_{l n} u_{n}\right)
$$

from $\quad \mathrm{W}=\frac{E^{2}+H^{2}}{8 \pi} \quad$ and $\quad \mathbf{P}^{(e m)}=\frac{\mathbf{E} \times \mathbf{H}}{4 \pi c} \quad$ with $\boldsymbol{u}_{\boldsymbol{k}}=\boldsymbol{\gamma} \mathbf{v}_{\boldsymbol{k}} \cdot \mathrm{T}_{\mathrm{ik}}$ is the usual energy-momentum tensor [1]. See Appendix A.

$$
T_{i k}=\frac{-1}{4 \pi}\left(F_{i l} F_{l k}+\frac{1}{4} \delta_{i k} F_{l m}^{2}\right)
$$

and $\mathrm{F}_{\mathrm{ik}}$ is the standard electromagnetic field tensor [1].

With assumption 5, the four-vector for current is

$$
J_{k}=\rho \mathrm{v}_{k}=\rho_{o} u_{k}, \mathrm{k}=1,2,3,4 .
$$

Define $a_{k} \equiv \mathrm{F}_{l k}^{l} \cdot \rho_{\mathrm{o}}$ is the covariant charge density. The covariant forms for Maxwell's equations are

$$
a_{k}=4 \pi J_{k} / c=4 \pi \rho_{o} u_{k} / c
$$

and

$$
F_{k m}^{l}+F_{l k}^{m}+F_{m l}^{k}=0 \text {. }
$$

Because $u_{k}^{2}=-c^{2}$ and because the fields are differentiable, (7) can be recast in the equivalent forms

$$
\rho_{o}= \pm i \sqrt{\frac{a_{m}^{2}}{4 \pi}}, u_{k}=\frac{ \pm i c a_{k}}{\sqrt{a_{m}^{2}}} \mathrm{k}, \mathrm{m}=1,2,3,4 .
$$

(signs correlate). Equation (8) implies that

$$
F_{l k}=A_{k}^{l}-A_{l}^{k}
$$

where

$$
\left(A_{k}\right)=\left(A_{1}, A_{2}, A_{3}, A_{4}\right)=\left(A_{x}, A_{y}, A_{z}, i \phi\right)
$$

is the covariant four-vector for potential.

$$
a_{k} \equiv F_{l k}^{l}=A_{k}^{l l}-A_{l}^{k l},
$$

The four-vector for

$$
\mathbf{f}=\gamma(\mathbf{E}+\vec{\beta} \times \mathbf{H}), \quad \mathrm{f}_{4}=i \vec{\beta} \cdot \mathbf{E}
$$

is

$$
\mathrm{f}_{k}=F_{k l} u_{l} / c .
$$

\section{Derivation of the Fundamental Equations}

We suppose that the Euler-Lagrange equations obtained from a variational principle

$$
\delta \int \operatorname{Ld} \Omega=0, d \Omega=d x_{1} d x_{2} d x_{3} d x_{4}
$$

provide physically meaningful "equations of motion", where $\mathrm{L}$ is a suitable Lagrange density and we alter the usual "action" for electromagnetic fields and particle motion $[1,3]$

$$
I=-\sum m c \int \sqrt{1-\beta^{2}} d t-\frac{i}{4 \pi c^{2}} \int J_{k} A_{k} d \Omega+\frac{i}{16 \pi c} \int F_{j k}^{2} d \Omega
$$

by replacing the first term with an invariant term appropriate to continuous fields. Let

$$
-\sum m c \int \sqrt{1-\beta^{2}} d t \rightarrow-i \int \mu c d \Omega
$$

so that

$$
I=-i \int \mu c d \Omega+\frac{i}{4 \pi c} \int a_{k} A_{k} d \Omega+\frac{i}{16 \pi c} \int F_{j k}^{2} d \Omega
$$

Taking $\mu$ from (3) and $T_{i k}$ from (5), one eventually arrives at the result (Appendix A)

$$
\begin{gathered}
I=\int L\left(A_{l}, A_{l}^{j}, A_{l}^{j k}\right) d x_{1} d x_{2} d x_{3} d x_{4} \\
L=\frac{1}{4 \pi c}\left(-F_{j l} F_{l k} \frac{a_{k} a_{j}}{a_{m}^{2}}+a_{k} A_{k}\right)
\end{gathered}
$$

where the indices are 1,2,3,4. A second-order variation $\delta \mathrm{I}=$ 0 on the field variables yields the Euler-Lagrange equations $[2,5]$

$$
\frac{\partial L}{\partial A_{k}}-\frac{\partial}{\partial x_{n}} \frac{\partial L}{\partial A_{k}^{n}}+\frac{\partial^{2}}{\partial x_{n} \partial x_{i}} \frac{\partial L}{\partial A_{k}^{n i}}=0
$$

Straightforward mathematical manipulations (Appendix A) yield four coupled, non-linear partial differential equations that are valid in and only in regions where $\rho \neq 0$.

$$
\frac{1}{2} \varepsilon_{n k l m} \varepsilon_{l m i j} \frac{\partial}{\partial x_{n}}\left[\frac{\partial}{\partial x_{i}}\left(\frac{c \zeta_{j}}{\rho_{O}}\right)-\frac{1}{c} \mathrm{f}_{j} u_{i}\right]=0
$$


where $\boldsymbol{\varepsilon}_{\boldsymbol{n} k \boldsymbol{d} \boldsymbol{m}}$ is the purely antisymmetric Levi-Civita tensor. Equation (19) will be satisfied if

$$
\frac{\partial}{\partial x_{l}}\left(\frac{c \zeta_{j}}{\rho_{O}}\right)=\mathrm{f}_{j} \frac{u_{l}}{c} \quad l \neq \mathrm{j}
$$

This equation is a $4 \times 4$ matrix of twelve independent equations where the entries with $l=\mathrm{j}$ are identically zero. Equation (20) is valid in regions where $\rho \neq 0$, and Maxwell's equations $\left(a_{m}=0\right)$ hold if $\rho=0$. The equations that explicitly involve time $\mathrm{t}(l=4, \mathrm{j}=1,2,3)$ will be used in what follows here.

$$
\frac{\partial}{\partial t}\left(\frac{\zeta_{j}}{\rho_{O}}\right)=-\gamma \mathrm{f}_{j}
$$

\section{Motion of Charged Bodies}

If $\rho_{\mathrm{o}}$ does not depend explicitly on time $t$, then (21) is

$$
\frac{\partial \zeta_{j}}{\partial t}=-\gamma \rho_{O} \mathrm{f}_{j}=-\rho \mathrm{f}_{j} \mathrm{j}=1,2,3
$$

Integrate over a volume $\mathrm{V}=\mathrm{V}_{\mathrm{o}}+\mathrm{V}_{\mathrm{i}}$. See Fig. 1 .

$$
\int_{V_{o}} \frac{\partial \zeta_{j}}{\partial t} d V+\int_{V_{i}} \frac{\partial \zeta_{j}}{\partial t} d V=-\int_{V_{i}} \rho \mathrm{f}_{j} d V
$$

The deformation of the charged volume when the body is accelerated is taken into account below.

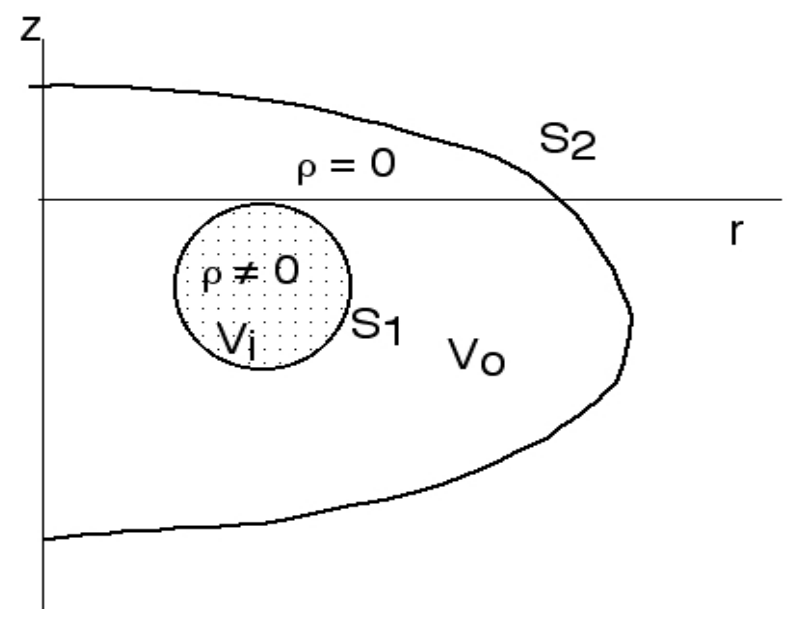

Figure 1. $S_{1}$ surrounds $V_{i}$, and $V_{o}$ is bounded by surfaces $S_{1}$ and $\mathrm{S}_{2} \cdot \mathrm{V}=\mathrm{V}_{\mathrm{i}}+\mathrm{V}_{\mathrm{o}}$.

Apply

$$
\frac{d}{d t} \int_{V(l)} F d V=\int_{V(t)} \frac{\partial}{\partial t} F d V+\oint_{S(t)} F \mathbf{v}_{S} \cdot \hat{\mathbf{n}} d S
$$

where $\mathrm{S}(\mathrm{t})$ surrounds $\mathrm{V}(\mathrm{t})$ and $\mathbf{v}_{\mathrm{S}}$ is the velocity of the surface. Equation (23) is then

$$
\begin{aligned}
& \frac{d}{d t} \int_{V_{0}} \zeta_{j} d V-\oint_{S_{1}} \zeta_{j} \mathbf{v} \cdot \hat{\mathbf{n}} d S-\oint_{S_{2}} \zeta_{j} \mathbf{v} \cdot \hat{\mathbf{n}} d S+ \\
& +\frac{d}{d t} \int_{V_{i}} \zeta_{j} d V-\oint_{S_{1}} \zeta_{j} \mathbf{v} \cdot \hat{\mathbf{n}} d S=-\int_{V_{i}} \rho \mathbf{f}_{j} d V
\end{aligned}
$$

where $\mathbf{v}$ is understood to be the velocity with which the particular surface $S_{1}$ or $S_{2}$ moves. The integrals over $S_{1}$ cancel because of the opposite directions of the normals and because the fields are continuous across $\mathrm{S}_{1}$, so

$$
\frac{d}{d t} \int_{V_{o}} \zeta_{j} d V+\frac{d}{d t} \int_{V_{i}} \zeta_{j} d V-\oint_{S_{2}} \zeta_{j} \mathbf{v} \cdot \hat{\mathbf{n}} d S=-\int_{V_{i}} \rho \mathrm{f}_{j} d V
$$

If the outer surface $\mathrm{S}_{2}$ is taken to be fixed in time, then

$$
\frac{d}{d t} \int_{V_{o}} \zeta_{j} d V+\frac{d}{d t} \int_{V_{i}} \zeta_{j} d V=-\int_{V_{i}} \rho \mathrm{f}_{j} d V
$$

If $\mathrm{S}_{2}$ is fixed at an arbitrarily large distance, the momentum $\mathrm{P}_{j}^{(\mathrm{em})}$ of a moving body is given by

$$
\begin{gathered}
\mathrm{P}_{j}^{(e m)}=-\int_{V_{i}}\left(\mu u_{j}+\frac{1}{c^{2}} T_{j n} u_{n}\right) d V-\int_{V_{o}}\left(\mu u_{j}+\frac{1}{c^{2}} T_{j n} u_{n}\right) d V \\
\mathrm{j}=1,2,3 \text { and } \mathrm{n}=1,2,3,4 .
\end{gathered}
$$

Set

$$
u_{j}=U_{j}+u_{j}^{\prime}
$$

where $U_{j}$ is the velocity of the body's center of inertia. If a charged body is moving with velocity $U_{j}$, then in regions where $\rho=0$, the velocity of the fields associated with that body is $\mathrm{U}_{\mathrm{j}}$.

If the linear momentum of a body at rest, i.e., $\mathrm{U}_{\mathrm{k}}=0$, is to be zero, then we must have

$$
\int_{V_{i}}\left(\mu u_{j}^{\prime}+\frac{1}{c^{2}} T_{j n} u_{n}^{\prime}\right) d V+\frac{\mathrm{U}_{4}}{c^{2}} \int_{V_{i}+V_{o}} T_{j 4} d V=0
$$

in which case, the three-momentum of a moving body is

$$
\mathrm{P}_{\mathrm{j}}^{(e m)}=-\mathrm{U}_{\mathrm{j}} \int_{V_{o}+V_{i}} \mu d V-\frac{\mathrm{U}_{k}}{c^{2}} \int_{V_{o}+V_{i}} T_{j k} d V
$$

$\mathrm{j}, \mathrm{k}=1,2,3$, or equivalently,

$$
\begin{gathered}
\mathrm{P}_{\mathrm{j}}^{(e m)}=-\mathrm{MU}_{\mathrm{j}}-\frac{\mathrm{U}_{k}}{c^{2}} \int_{V_{o}+V_{i}} T_{j k} d V \\
M=\int_{V_{\boldsymbol{i}}+V_{o}} \mu d V
\end{gathered}
$$

Since $\mu$ is invariant under Lorentz transformations, $M$ will be invariant when integrated over the proper volume 
(invariance means that if frames 1 and 2 are moving with relative velocity $\beta_{12}$, then $\int \mu_{1} d t_{1} d V_{1}=\int \mu_{2} d t_{2} d V_{2}=\int \mu_{2} \gamma_{12} d V_{2} d t_{1}$.) Hence, the temporal derivative of $\mathrm{P}_{j}^{(e m)}$ gives

$$
\frac{d\left(\mathrm{MU}_{\mathrm{j}}\right)}{d t}+\frac{1}{c^{2}} \frac{d}{d t}\left(\mathrm{U}_{\mathrm{k}} \int_{V_{o}+V_{i}} T_{j k} d V\right)=\int_{V_{i}} \rho \mathrm{f}_{\mathrm{j}} d V
$$

$\mathrm{j}, \mathrm{k}=1,2,3$. Thus, the temporal rate of change of the momentum of an electromagnetic body is determined by the Lorentz force. In this equation, $M$ includes the equivalent mass of all the fields. Since M is invariant, we can calculate $\mathrm{M}$ when the body is at rest.

$$
M=\frac{1}{c^{4}} \int_{V_{i}} T_{i k} u_{i}^{\prime \prime} u_{k}^{\prime \prime} d V+\frac{1}{8 \pi c^{2}} \int_{V_{a}}\left(E^{2}+H^{2}\right) d V
$$

where the double primes designate the velocities when the system is at rest.

\section{Static Solutions}

\section{A. Static equations}

Static (time-independent) solutions require that

$$
\mathbf{f}=\gamma(\mathbf{E}+\vec{\beta} \times \mathbf{H})=0
$$

See (22). Solution of (36) and Maxwell's equations will yield contained and stable charge distributions.

$$
\begin{gathered}
\nabla \times \mathbf{E}=\mathbf{0} \\
\nabla \times \mathbf{H}=\vec{\beta} \nabla \cdot \mathbf{E} \\
\nabla \cdot \mathbf{H}=0
\end{gathered}
$$

Solve these equations in cylindrical symmetry $(r, z . \theta)$ with $\mathrm{H}_{\theta}=\mathrm{E}_{\theta}=0$, no functional dependence on $\theta$, and $\vec{\beta}=\hat{\theta} \beta(r, z)$ :

$$
\begin{gathered}
\mathbf{H}(\mathrm{r} \cdot \mathrm{z})=\hat{\mathbf{r}} \mathrm{H}_{\mathrm{r}}+\hat{\mathbf{z}} \mathrm{H}_{\mathrm{z}} \\
\mathbf{E}(r \cdot z)=\hat{\mathbf{r}} E_{r}+\hat{\mathbf{z}} E_{z}=-\nabla \phi
\end{gathered}
$$

Equation (36) then reduces to

$$
\mathrm{E}_{\mathrm{r}}=-\boldsymbol{\beta} \mathrm{H}_{\mathrm{z}} \text { and } \mathrm{E}_{\mathrm{z}}=\boldsymbol{\beta} \mathrm{H}_{\mathrm{r}}
$$

Equations (37), (39), and (42) can be combined to give $\frac{\partial \phi}{\partial z} \frac{\partial}{\partial r}\left(\frac{r}{\beta}\right)-\frac{\partial \phi}{\partial r} \frac{\partial}{\partial z}\left(\frac{r}{\beta}\right)=0$, which implies that either $\phi=\phi(\mathrm{r} / \beta)$ or $\beta=\mathrm{Cr}$, where $\phi$ is any function and $\mathrm{C}$ is a constant. Use (42) to eliminate $\mathrm{H}$ in (38) and use $\mathrm{E}=-\nabla \phi$ to get

$$
\left(1-\beta^{2}\right)\left(\frac{\partial^{2} \phi}{\partial r^{2}}+\frac{\partial^{2} \phi}{\partial z^{2}}\right)-\frac{1}{\beta}\left(\frac{\partial \beta}{\partial z} \frac{\partial \phi}{\partial z}+\frac{\partial \beta}{\partial r} \frac{\partial \phi}{\partial r}\right)-\frac{\beta^{2}}{r} \frac{\partial \phi}{\partial r}=0
$$

Put $\beta=\mathrm{Cr}$ in (43) and obtain a linear P.D.E.

$$
\left(1-\beta^{2}\right)\left(\frac{\partial^{2} \phi}{\partial \beta^{2}}+\frac{1}{C^{2}} \frac{\partial^{2} \phi}{\partial z^{2}}\right)-\frac{1+\beta^{2}}{\beta} \frac{\partial \phi}{\partial \beta}=0
$$

\section{B. Some static solutions}

There are many approaches to solving (44). Two series solutions and one approximate solution are presented below.

The condition given in (30) is satisfied for the cylindrical static solutions found here, and $f_{j}$ in (34) is equal to the externally impressed fields. In addition:

(1) There will be an equipotential surface $(\phi=$ const $)$ that bounds the charged region of a static body.

(2) If the electric fields of a charged body are to originate entirely from the charges within the body and if the body is to have mirror symmetry in the $\mathrm{z}$ $=0$ plane, then the electric potential must be continuous and $\mathrm{E}_{\mathrm{z}}=0$ when $\mathrm{z}=0$. That is,

$$
\left(\frac{\partial \phi}{\partial z}\right)_{z=0}=0 .
$$

Insert $\phi(\beta, \zeta)=\mathrm{R}(\beta) \mathrm{Z}(\zeta)$ into (44) with $\zeta=\mathrm{Cz}$ and

$$
\mathrm{Z}(\zeta)=\cos (\mathrm{kz})=\cos (\alpha \zeta), \alpha=\mathrm{k} / \mathrm{C}
$$

The resulting O.D.E. is

$$
\beta\left(1-\beta^{2}\right)\left(\frac{\partial^{2} R}{\partial \beta^{2}}-\alpha^{2} R\right)-\left(1+\beta^{2}\right) \frac{\partial R}{\partial \beta}=0
$$

\section{Series expansion around $\beta \approx 0$}

Equation (47) can be solved in many ways, e.g., set

$$
R(\beta)=\sum_{n=1}^{\infty} a_{n} \beta^{n}
$$

to obtain the recursion relation

$$
n(n+2) a_{n+2}-\left(n^{2}+\alpha^{2}\right) a_{n}+\alpha^{2} \mathrm{a}_{\mathrm{n}-2}=0
$$

Equation (49) gives two independent solutions of (47), and the first three terms of these series are given below.

$$
\begin{aligned}
R_{o}(\beta, \alpha) & =a_{1}\left[\beta+\frac{1+\alpha^{2}}{3} \beta^{3}+\frac{1}{45}\left(9+7 \alpha^{2}+\alpha^{4}\right) \beta^{5}+\ldots\right] . \\
& R_{e}(\beta, \alpha)=\mathbf{a}_{2}\left[\beta^{2}+\frac{4+\alpha^{2}}{8} \beta^{4}+\right. \\
& \left.\left.+\frac{1}{192}\left(\left(16+\alpha^{2}\right)\left(4+\alpha^{2}\right)-8 \alpha^{2}\right) \beta^{6}+\ldots\right)\right]
\end{aligned}
$$

A general solution of (44) with $\mathrm{Z}(\mathrm{z})=\cos (\mathrm{kz})$ is $\phi(\alpha, \beta, \zeta)=\sum_{j} A_{j} \operatorname{Cos}\left(\alpha_{j} \zeta\right)\left(R_{o}\left(\alpha_{j}, \beta\right)+\eta_{j} R_{e}\left(\alpha_{j}, \beta\right)\right)+\kappa$ 
where $A_{j}, \eta_{j}$, and $\kappa$ are arbitrary constants. $\zeta=\mathrm{Cz}, \beta=\mathrm{Cr}$, and the $\alpha_{\mathrm{j}}$ are values of $\alpha_{\mathrm{j}}=(\mathrm{k} / \mathrm{C})_{\mathrm{j}}$ appropriate to the problem. Figure 2 shows a particular solution when $\alpha=1$ and

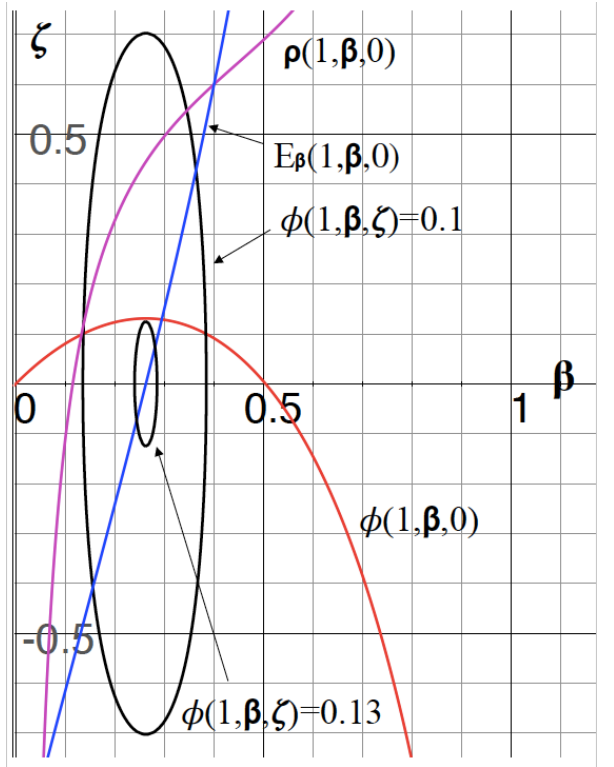

Figure 2. Depiction of $\phi$ from (53), associated electric field $E_{\beta}$ and charge density $\rho$ versus $\beta$. Equipotential curves defining static charged bodies are shown for $\phi=0.13$ and $0.1 . \alpha=\mathrm{k} / \mathrm{C}=1$, with ordinate and abscissa having the same scale. The ordinate scale is arbitrary for $E_{\beta}$ and $\rho$. The series representation has been expanded to $\beta^{14}$.

$$
\phi(1, \beta, \zeta)=\left[R_{o}(\beta, 1)-2 R_{e}(\beta, 1)\right] \cos (\zeta) .
$$

To find charged regions enclosed by an equipotential, we set $\phi(\alpha, \beta, \zeta)=$ constant. The boundaries of the spinning bodies are defined by rotating the equipotential contours shown around the $\zeta$ (or $\mathrm{z}$ ) axis. The resultant spinning ring of charge has no temporal dependence and thus represents a stable charged body.

Figure 3 depicts a generic shape, a spinning ring, applicable to all of the bodies derived here. The field outside the charged ring is obtained by solving Maxwell's equations in free space and matching the fields and potentials to those on the surface of the ring.

Persons familiar with forces and particles will ask what holds the charged shape together. Equation (36) shows that the electromagnetic forces balance out. Combining (4) and (36) shows that the mass and fields exchange momentum in a way that maintains a net momentum of zero within the spinning charged ring. The result is a static body.

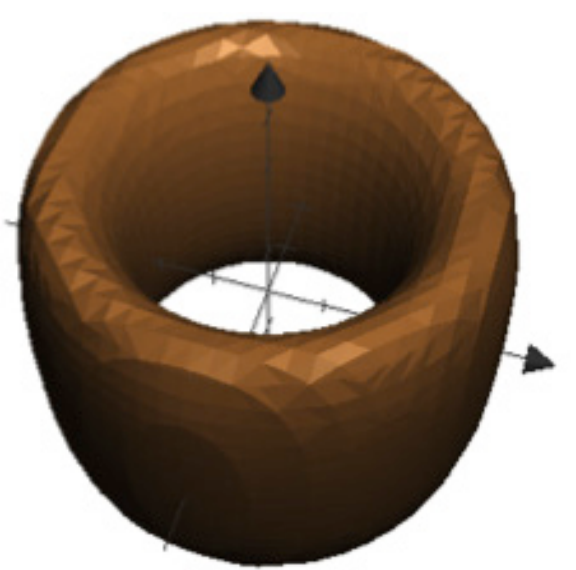

Figure 3. Generic depiction of spinning hollow cylindrical bodies. These shapes are generated by rotating the equipotentials shown in the $\beta \zeta$ plane about the $\zeta$ axis.

\section{Series Expansion Around $\beta \approx 1$}

Let $\mathrm{R}$ be a function of $\mathrm{h}=1-\beta^{2}$. Then (47) becomes

$$
(1-h)\left(4 h R^{\prime \prime}+2 R^{\prime}\right)-\alpha^{2} h R=0
$$

where the primes denote derivatives with respect to $h$. If

$$
R(h)=h^{s} \sum_{n=0}^{\infty} a_{n} h^{n}
$$

The indicial equation is $\mathrm{s}(2 \mathrm{~s}-1)=0 ; \mathrm{s}=0$ gives

$$
a_{n+1}=\frac{2 n(2 n-1) a_{n}+\alpha^{2} a_{n-1}}{(2 n+2)(2 n+1)}
$$

and corresponding series

$$
R_{o}(h)=1+\frac{\alpha^{2}}{12} h^{2}+\frac{\alpha^{2}}{30} h^{3}+\frac{\alpha^{2}}{56}\left(1+\frac{\alpha^{2}}{12}\right) h^{4}+\ldots .
$$

For $\mathrm{s}=1 / 2$, the equations are, with $\mathrm{c}_{\mathrm{n}}$ replacing $\mathrm{a}_{\mathrm{n}}$,

$$
c_{n+1}=\frac{2 n(2 n+1) c_{n}+\alpha^{2} c_{n-1}}{(2 n+2)(2 n+3)}
$$

and

$$
R_{1 / 2}(h)=h^{1 / 2}\left[1+\frac{\alpha^{2}}{20} h^{2}+\frac{\alpha^{2}}{42} h^{3}+\frac{\alpha^{2}}{72}\left(1+\frac{\alpha^{2}}{20}\right) h^{4}+\ldots\right]
$$

If $|\mathrm{h}|<1$, these series converge for all finite values of $\alpha^{2}$; however, when $\alpha^{2}>1$, convergence is slow. The general solution of (44) is

$$
\phi(\alpha, h, \zeta)=\sum_{j} A_{j} \operatorname{Cos}\left(\alpha_{j} \zeta\right)\left(R_{0}\left(\alpha_{j}, h\right)+\eta_{j} R_{1 / 2}\left(\alpha_{j}, h\right)\right)+\kappa
$$


Curves for $\phi(7, h, 0)=1490 \mathrm{R}_{0}-2888.6 \mathrm{R}_{1 / 2}$ are shown in Fig. 4, with series terms to $\mathrm{h}^{20}$. Ordinate and abscissa are scaled properly for $\phi=-1.5$, but $E_{\beta}$ has been multiplied by $4 \pi / 2000$ and $\rho$ by $4 \pi / 10^{4}$. The axis of rotation is at $h=1$, off to the right. The abscissa/ordinate scale ratio is 125 , so the height of each equipotential surface depicted is physically $\approx 11$ times its width. $\phi$ maximizes at $\mathrm{h} \approx 0.511$, i.e., $\beta \approx 0.699$.

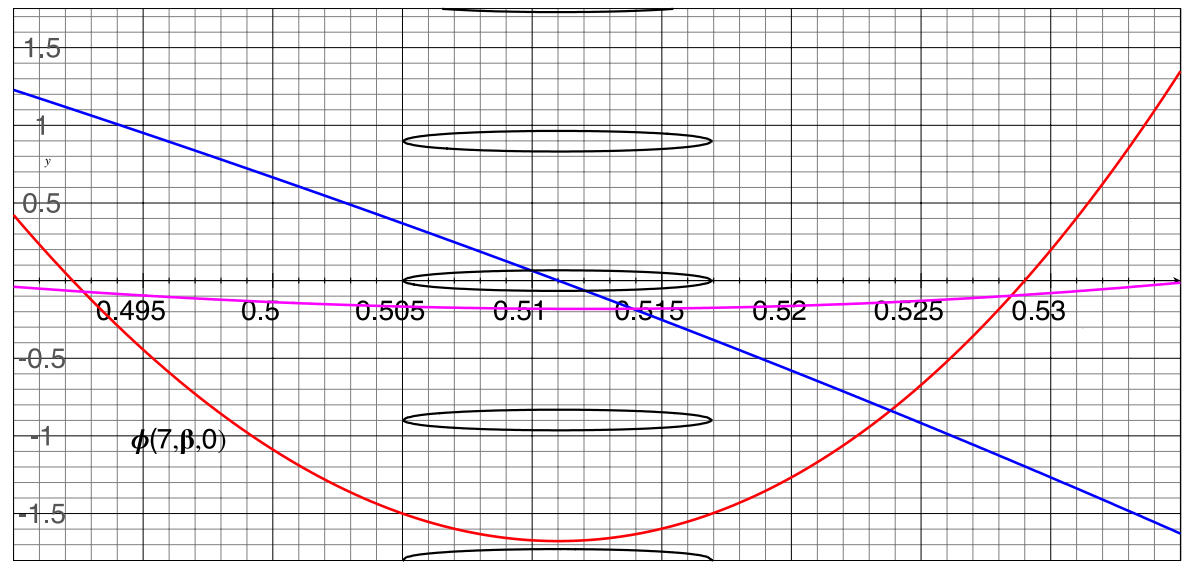

Figure 4. Depiction of $\phi(7, h, 0)=1490 R_{0}-2888.6 R_{1 / 2}, E_{\beta}$, and $\rho$ versus $h$ for $\alpha=7$. Curves for $\left(1490 R_{0}-2888.6 R_{1 / 2}\right) \cos (7 \zeta)=-1.5$ are also shown.

\section{Approximate Solution When $\varepsilon=1-\beta<1$}

Rewrite (47) with $\mathrm{R}$ as a function of $1-\beta=\varepsilon<<1$. Then, approximately, $1-\beta^{2} \approx 2 \varepsilon, 1+\beta^{2} \approx 2(1-\varepsilon)$, and

$$
\varepsilon\left(\frac{d^{2} R}{d \varepsilon^{2}}-\alpha^{2} R\right)+\frac{d R}{d \varepsilon} \approx 0
$$

with solution $\mathrm{R}=\mathrm{Z}_{\mathrm{o}}(\alpha \varepsilon)$ where $\mathrm{Z}_{\mathrm{o}}$ is a modified Bessel function $\left[\mathrm{I}_{0}(\alpha \varepsilon)\right.$ or $\left.\mathrm{K}_{\mathrm{o}}(\alpha \varepsilon)\right]$ of order zero. As before, $\zeta=\mathrm{Cz}$ and $\beta=\mathrm{Cr}$. A general solution is now given by

$$
\phi(\varepsilon, \zeta)=\kappa+\sum_{j} A_{j} \operatorname{Cos}\left(\alpha_{j} \zeta\right)\left(I_{o}\left(\alpha_{j} \varepsilon\right)+B_{j} K_{o}\left(\alpha_{j} \varepsilon\right)\right)
$$

where $\kappa, A_{j}$, and $B_{j}$ are arbitrary constants. As a simple example, take $\alpha_{0}=370000$ and $\alpha_{1}=1, \mathrm{~A}_{\mathrm{o}}=-0.001, \mathrm{~B}_{\mathrm{o}}=$ $5 \times 10^{3}, A_{1}=0.2, \kappa=-0.1$, and all other terms equal to zero.

$$
\begin{aligned}
& \phi(\varepsilon, \zeta)= \\
& -0.1+\left[-0.001 I_{0}(370000 \varepsilon)-5 K_{0}(370000 \varepsilon)\right] \operatorname{Cos}(37000 \zeta)(62) \\
& +0.2 I_{0}(\varepsilon) \operatorname{Cos}(\zeta)
\end{aligned}
$$

Figure 5 shows the potential, electric field, charge density, and two closed equipotential curves in the $\beta \zeta$-plane for the potential given by (62). The constant $\kappa=-0.1$ is chosen to make $\rho$ and $\phi$ zero at approximately the same value of $\varepsilon$. If $\phi$ and the equipotentials are shown on the proper scale, then $E_{\beta}$ should be multiplied by 72000 and $\rho$ should be multiplied by $10^{11} / 4 \pi$. The axis of rotation is at $\varepsilon$ $=1$, far to the right in the figure.

\section{Extrinsic properties of static charged bodies}

The angular momentum density is an antisymmetric tensor

$$
l_{j k}=x_{j} \zeta_{k}-x_{k} \zeta_{j}
$$

The total angular momentum of a charged, static body is

$$
L_{j k}=\int_{V_{i}+V_{0}}\left(x_{j} \zeta_{k}-x_{k} \zeta_{j}\right) d V=\int_{V_{0}}\left(x_{j} \zeta_{k}-x_{k} \zeta_{j}\right) d V
$$

because $\zeta_{\mathrm{j}}=0$ in $\mathrm{V}_{\mathrm{i}}$. The angular momentum inherent to a stationary charged body will then be given by

$$
\mathbf{L}=\frac{1}{4 \pi c} \int_{V_{0}} \mathbf{r} \times(\mathbf{E} \times \mathbf{H}) d V
$$

The magnitude of $\mathbf{L}$ and its projection along one axis are invariant under Lorentz transforms.

The magnetic moment of the body is, in Gaussian units,

$$
m_{j k}=\frac{1}{2 c} \int_{V_{i}}\left(x_{j} J_{k}-x_{k} J_{j}\right) d V=\frac{1}{2 c} \int_{V_{i}} \rho_{o}\left(x_{j} u_{k}^{\prime \prime}-x_{k} u_{j}^{\prime \prime}\right) d V
$$

Equivalently,

$$
m_{j k}=-\frac{1}{8 \pi} \int_{V_{i}}\left(x_{j} a_{k}^{\prime \prime}-x_{k} a_{j}^{\prime \prime}\right) d V
$$

The double primes are reminders that the velocities and fields are those of the body at rest. The connection between the charged body's angular momentum and magnetic moment is implicit because the integrals are over different volumes and the magnetic fields in $\mathrm{V}_{\mathrm{o}}$ are generated by the charge motions in $\mathrm{V}_{\mathrm{i}}$.

The total charge on a static body will be

$$
Q=\int_{V_{i}} \rho_{O} d V= \pm \frac{i}{4 \pi} \int_{V_{i}} \sqrt{a_{m}^{2}} d V
$$


where $a_{k}=F_{l k}^{l}$. Because $\rho_{\mathrm{o}}$ and $a_{m}^{2}$ are invariant, Q will also be invariant when integrated over the proper volume in different moving frames.

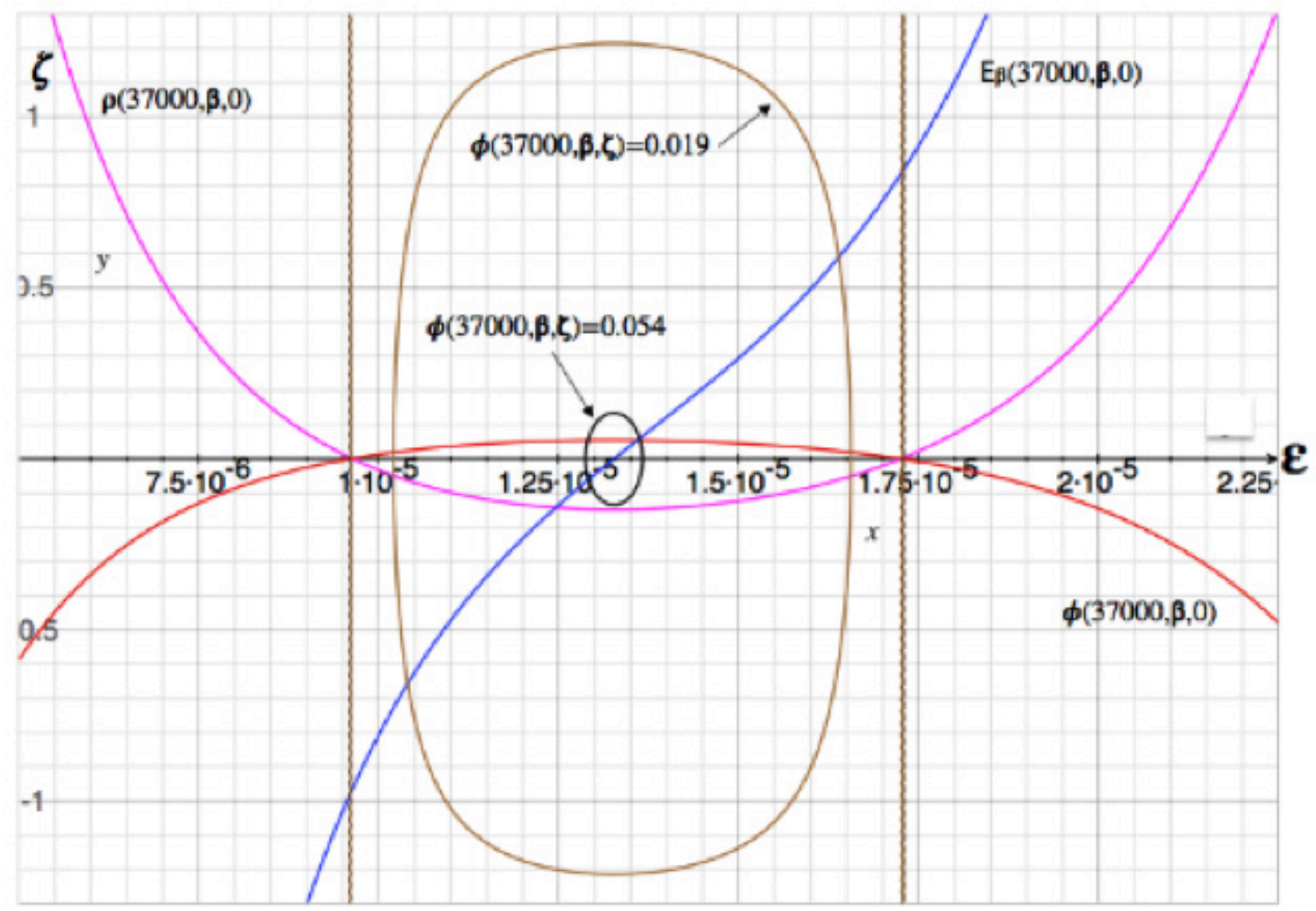

Figure. 5. Potential, radial electric field, charge density, and equipotential curves for the potential given in (62) plotted versus $\varepsilon=1-\beta$, $\beta>0$.

\section{Fundamental Bodies in This Field Electrodynamics}

In principal, this classical field theory of electrodynamics can represent all charged bodies (electrons, protons, neutrons, etc.). The theory may provide a description; however, unlike quantum mechanics, it has not provided a unique catalogue of types or states.

The magnetic moment $\mathbf{m}$ and quadrupole moment $\mathrm{D}$ can easily be adequately (not accurately) estimated for a body with given charge. The spin and mass require machine computations and will be reported in a future paper.

Consider the electron as an example. The magnetic moment for these spinning charged rings is given by (66), which is approximately

$$
m \approx \frac{1}{2 c} 2 \pi r_{o} A \rho r_{o} \mathrm{v}_{o} y_{o} \approx \frac{1}{2 c} Q r_{o} \mathrm{v}_{o} \gamma_{o} \approx \frac{1}{2} Q r_{o} \beta_{o} \gamma_{o}
$$

where $r_{0}, \beta_{0}, \gamma_{0}$ are representative values within the charged region. The magnetic moment of the electron is (in Gaussian units) $9.284 \times 10^{-21} \mathrm{erg} / \mathrm{G}$, the charge on the electron is $\mathrm{Q}=4.803 \times 10^{-10}$ statcoul, and the speed of light, $\mathrm{c}=2.99792 \times 10^{10} \mathrm{~cm} / \mathrm{s}$. Thus,

$$
r_{O} \beta_{O} \gamma_{O} \approx 3.866 \times 10^{-11} \mathrm{~cm}
$$

In Fig. 5, the larger contour corresponds to $\mathrm{D} \approx 0$ and $\beta_{\mathrm{o}}$ $\approx 1$. With $\beta \gamma \approx 193$, the ring's radius $\mathrm{r}_{\mathrm{o}} \approx 2 \times 10^{-13} \mathrm{~cm}$ and $\mathrm{C}$ $=\beta_{\mathrm{o}} / \mathrm{r}_{\mathrm{o}} \approx 5 \times 10^{12} \mathrm{~cm}^{-1}$. The height and thickness of the ring are, respectively, $\approx 4.8 \times 10^{-13}$ and $1.24 \times 10^{-18} \mathrm{~cm}$.

In Fig. 2, $\mathrm{D}=0$ for an equipotential contour between $\phi=$ 0.1 and $0.13 ; \beta_{\mathrm{o}} \approx 0.26, \beta_{\mathrm{o}} \gamma_{\mathrm{o}} \approx 0.269, \mathrm{r}_{\mathrm{o}} \approx 1.44 \times 10^{-10} \mathrm{~cm}$, and $\mathrm{C}=\beta_{\mathrm{o}} / \mathrm{r}_{\mathrm{o}}=1.81 \times 10^{9} \mathrm{~cm}^{-1}$. The thickness and height of the smaller annulus in Fig. 2 would then be $\Delta \mathrm{r}_{\mathrm{o}} \approx 2.5 \times 10^{-11} \mathrm{~cm}$ and $1.27 \times 10^{-10} \mathrm{~cm}$, respectively. This solution looks somewhat similar to a classical version of zitterbewegung.

These examples strongly indicate that the electron can be represented by one of these spinning rings. There are six parameters $\left(r_{o}, \alpha, A_{j}, \kappa, C\right.$, and the value of the surrounding equipotential) to reproduce, in principle, the mass, charge, spin, quadrupole moment $\mathrm{D}$, and magnetic moment of an electron, and there is even more flexibility if series having different $\alpha$ are summed.

Scattering experiments indicate that the proton's structure is complicated, so it may require a few spinning rings to represent the proton. For a single small ring of approximately the measured size of the proton, $\mathrm{r}_{0} \approx$ $8.7 \times 10^{-14} \mathrm{~cm}$, as in (69), with a magnetic moment $\approx$ 
$1.41 \times 10^{-14} \mathrm{erg} / \mathrm{G}, r_{O} \beta_{O} \gamma_{O} \approx 5.82 \times 10^{-14} \mathrm{~cm}$, gives $\beta \approx$ 0.309 . Rough estimates of the spin and mass suggest that there are charge distributions circulating at radii considerably smaller than $8.7 \times 10^{-14} \mathrm{~cm}$. If there were more rings, the charges on each ring would add to give the charge of the proton and the magnetic moments of each ring would add to give the proton magnetic moment, but the spin and mass must be calculated in detail from the resultant fields throughout the whole of space. These latter calculations cannot be done conveniently by hand.

\section{Comparison with Other Work}

The author is unaware of any previous second-order variational theory of classical electrodynamics or of any theoretical body comprising charge of a single sign and stabilized by electromagnetic interactions. There are many models of the electron, so we will compare with those.

The classical electron derived here is a spinning ring, not any of the toroids or helical entities postulated in earlier models of an electron [7-12]. Most models of the electron are ad hoc and focus on the connection between the model and quantum mechanics. Most, but not all, of these treatments are dualistic, with point particles and fields $[4,12]$. Some modifications of classical electrodynamics invoke two or more temporal or physical scales or modifications of Maxwell's equations or of the Lorentz force, sometimes involving gravity $[4,8,13,18]$.

\section{Future Work}

Not all of the implications of a non-dualistic field theory of electrodynamics are addressed here. A few obvious topics are:

- Computer calculations of the properties of these static bodies.

- $\quad$ Time dependence using all twelve equations (20).

- Radiation reaction can be described by (26) with (24) applied to $\left(A_{k}\right)$ in $(10)$.

- A systematic characterization of the solutions of (47).

- $\quad$ Solutions of the form $\phi=\phi(\beta / r)$.

- Scattering phenomena in very intimate collisions.

- Incorporation of field electrodynamics into general relativity.

\section{Summary}

This paper presents a reformulation of classical electrodynamics in which the well-known Lorentz force law emerges as a derived equation and the structure of static bodies comprising charge of one sign is obtained. These static bodies involve only electromagnetic interactions. In this theory, charge is always associated with motion. It is the motion of the charge that produces the fields that stabilize charged bodies.

The assertion [1] that point charges must be used classically because extended bodies would distort at high speeds has been shown to be invalid. Extended bodies do distort, but when the fields are continuous, bodies still move as predicted by the Lorentz force law.

Consider the electron, which in standard electromagnetic theory is an enigmatic point held together by an unknown force and having infinite mass and charge density with a spin and magnetic moment arising from some unknown source. In the present construct, the electron is a spinning ring of charge held together by its internal velocity and electromagnetic self-fields. Those fields and the corresponding external fields yield finite mass and charge densities, which can be integrated to give finite mass and charge. Magnetic moment results from the motion of charge, and spin arises from the electromagnetic field produced by the motion of the charge.

It has been no part of this paper to relate this theory to quantum mechanics; however, see de Wet [19]. Also, Simulik and Krivsky [16-18] report that their modification of Maxwell's equations reproduces some quantum results. The quantum properties of the electron arise because its de Broglie wavelength is much larger than its size and is virtually independent of its structure.

The discovery that small charged bodies require no new strong forces to hold them together classically and knowledge of the classical structure of fundamental bodies, such as the electron, may facilitate understanding of the quantum nature of the electron and other fundamental bodies. A "particle theory" based on a field theory of electrodynamics would differ significantly from one based on the dualistic concept of distinct fields and particles.

\section{Acknowledgements}

This work was supported in part by the Los Alamos National Laboratory, the University of Nottingham, and the Naval Postgraduate School. I am grateful for help and suggestions from David Book, Abraham Kadish, Richard Robiscoe, William C. Maier, Volodomir Simulik, and Alyssa Turner.

\section{Appendix A.}

\section{Derivation of (17) and (19)}

From (15)

$L=-\mu c+\frac{1}{4 \pi c} a_{n} A_{n}+\frac{1}{16 \pi c} F_{l m}^{2}$ 


$$
\begin{aligned}
& \mu=\frac{1}{c^{4}} T_{i k} u_{i} u_{k}=\frac{-1}{4 \pi c^{4}}\left(F_{i l} F_{l k}+\frac{1}{4} \delta_{i k} F_{l m}^{2}\right) u_{i} u_{k} \\
& u_{k}=\frac{ \pm i c a_{k}}{\sqrt{a_{m}^{2}}} \\
& \rho_{\boldsymbol{o}}= \pm i \frac{\sqrt{a_{m}^{2}}}{4 \pi} \\
& \mu=\frac{1}{4 \pi c^{2}}\left(F_{j l} F_{l k} \frac{a_{k} a_{j}}{a_{m}^{2}}+\frac{1}{4} F_{l m}^{2}\right) \\
& L=\frac{1}{4 \pi c}\left(-F_{j l} F_{l k} \frac{a_{k} a_{j}}{a_{m}^{2}}+a_{k} A_{k}\right)
\end{aligned}
$$

Set $L_{O}=F_{j l} F_{l k} \frac{a_{j} a_{k}}{a_{m}^{2}}$ and $L_{2}=a_{k} A_{k}$

$\frac{\partial L}{\partial A_{k}}=\frac{\partial L_{2}}{\partial A_{k}}=a_{k}$

$\frac{\partial L_{2}}{\partial A_{k}^{\alpha \beta}}=A_{n} \frac{\partial a_{n}}{\partial A_{k}^{\alpha \beta}}=A_{n} \frac{\partial}{\partial A_{k}^{\alpha \beta}}\left(A_{l}^{n l}-A_{n}^{l l}\right)=A_{\alpha} \delta_{\beta k}-A_{k} \delta_{\beta \alpha}$

$\frac{\partial}{\partial x_{\alpha} \partial x_{\beta}}\left(\frac{\partial L_{2}}{\partial A_{k}^{\alpha \beta}}\right)=-a_{k} \frac{\partial L_{2}}{\partial A_{k}^{\alpha}}=0$

$\frac{\partial L_{2}}{\partial A_{k}}+\frac{\partial}{\partial x_{\alpha} \partial x_{\beta}}\left(\frac{\partial L_{2}}{\partial A_{k}^{\alpha \beta}}\right)=0$

So, applying (18) to $\mathrm{L}_{2}$ yields identically zero, and the term $\mathrm{J}_{\mathrm{k}} \mathrm{A}_{\mathrm{k}}$ in (14) contributes nothing (!) to the equations obtained from a second-order variation of this $\mathrm{L}$.

$$
\begin{aligned}
& \frac{\partial L_{o}}{\partial A_{k}^{\alpha}}=\frac{\partial L_{o}}{\partial F_{i j}} \frac{\partial F_{i j}}{\partial A_{k}^{\alpha}} \\
& \frac{\partial F_{i j}}{\partial A_{k}^{\alpha}}=\frac{\partial}{\partial A_{k}^{\alpha}}\left(A_{j}^{i}-A_{i}^{j}\right)=\delta_{j k} \delta_{i \alpha}-\delta_{j \alpha} \delta_{k i} \\
& \frac{\partial L_{o}}{\partial F_{i j}}=\frac{a_{n}}{a_{m}^{2}}\left(a_{j} F_{n i}-F_{n j} a_{i}\right) \\
& \frac{\partial L_{o}}{\partial A_{k}^{\alpha}}=2 \omega_{\alpha k} \\
& \omega_{\alpha k}=\frac{a_{n}}{a_{m}^{2}}\left(a_{k} F_{n \alpha}-F_{n k} a_{\alpha}\right) \\
& \frac{\partial}{\partial x_{\alpha}} \frac{\partial L_{o}}{\partial A_{k}^{\alpha}}=2 \omega_{\alpha k}^{\alpha}
\end{aligned}
$$

$$
\begin{aligned}
& \frac{\partial L_{o}}{\partial A_{k}^{\alpha \beta}}=\frac{\partial L_{o}}{\partial a_{n}} \frac{\partial a_{n}}{\partial A_{k}^{\alpha \beta}} \\
& \frac{\partial L_{o}}{\partial a_{n}}=2 \frac{F_{l i} a_{i} a_{j}}{\left(a_{m}^{2}\right)^{2}}\left(a_{j} F_{n l}-a_{n} F_{j l}\right) \equiv 2 \Gamma_{n} \\
& \frac{\partial a_{n}}{\partial A_{k}^{\alpha \beta}}=\delta_{\alpha \beta} \delta_{k n}-\delta_{k \beta} \delta_{\alpha n} \\
& \frac{\partial L_{o}}{\partial A_{k}^{\alpha \beta}}=2\left(\Gamma_{k} \delta_{\alpha \beta}-\Gamma_{\alpha} \delta_{k \beta}\right) \\
& \frac{\partial}{\partial x_{\alpha} \partial x_{\beta}}\left(\frac{\partial L_{o}}{\partial A_{k}^{\alpha \beta}}\right)=2\left(\Gamma_{k}^{\alpha \alpha}-\Gamma_{\alpha}^{k \alpha}\right)
\end{aligned}
$$

Put these results into (18) and obtain $\frac{\partial}{\partial x_{\alpha}}\left(\omega_{\alpha k}+\Gamma_{\alpha}^{k}-\Gamma_{k}^{\alpha}\right)=0$

$\frac{\partial}{\partial x_{a}}\left\{\begin{array}{l}\frac{a_{n}}{a_{m}^{2}}\left(a_{k} F_{n a}-F_{n k} a_{a}\right)+\frac{\partial}{\partial x_{k}}\left[\frac{F_{l i} a_{i} a_{j}}{\left(a_{m}^{2}\right)^{2}}\left(a_{j} F_{a l}-a_{n} F_{j l}\right)\right]- \\ -\frac{\partial}{\partial x_{a}}\left[\frac{F_{l i} a_{i} a_{j}}{\left(a_{m}^{2}\right)^{2}}\left(a_{j} F_{k l}-a_{k} F_{j l}\right)\right]\end{array}\right\}=0$

$\zeta_{l}=\frac{1}{4 \pi c^{4}}\left[F_{n k} u_{n}\left(F_{l k} u_{j}-F_{j k} u_{l}\right) u_{j}\right]$

$\frac{\partial}{\partial x_{a}}\left\{\frac{u_{n}}{c^{2}}\left(u_{k} F_{n a}-F_{n k} u_{a}\right)+\left[\frac{\partial}{\partial x_{k}}\left(\frac{c \zeta_{a}}{\rho_{O}}\right)-\frac{\partial}{\partial x_{a}}\left(\frac{c \zeta_{k}}{\rho_{O}}\right)\right]\right\}=0$

$\left(\delta_{i a} \delta_{j k}-\delta_{j a} \delta_{k i}\right) \frac{\partial}{\partial x_{a}}\left[\frac{\partial}{\partial x_{i}}\left(\frac{c \zeta_{j}}{\rho_{O}}\right)+\frac{1}{c^{2}} F_{n j} u_{n} u_{i}\right]=0$

Equation (19) follows.

\section{Derivation of (3) and (4)}

Equation (3) is obtained directly by substituting (1) and (2) into $\mathrm{E}^{2}+\mathrm{H}^{2}$ and comparing it with the expanded version of (3). Equation (4) is inferred from the expression obtained by substituting (1) and (2) into ExH. The result is

$$
\begin{aligned}
& 4 \pi c^{4} \zeta_{l}=\frac{1}{2} \varepsilon_{l m p s} \varepsilon_{p s h g} F_{j r} F_{m} u_{k} u_{n} u_{m}= \\
& =\left(\delta_{k j} \delta_{j m}-\delta_{l j} \delta_{m k}\right) F_{j r} F_{m} u_{k} u_{n} u_{m}=F_{m} u_{n}\left(u_{l} u_{m} F_{m s}-u_{m}^{2} F_{b}\right)= \\
& =F_{n r} u_{n}\left(u_{m} F_{l r}-u_{l} F_{m r}\right) u_{m} \\
& \text { As } \beta->0, \quad\left\{\zeta_{l}\right\} \rightarrow\left\{\frac{\mathbf{E} \times \mathbf{H}}{4 \pi c}, 0\right\} .
\end{aligned}
$$




\section{REFERENCES}

[1] L.D. Landau and E. M. Lifshitz, The Classical Theory of Fields, 2nd Edition, Trans by M. Hammermesh, Addison-Wesley, Reading, Mass, 1962, pp. 48, 67, 80, 92.

[2] A. O. Barut, Electrodynamics and Classical Theory of Fields and Particle, Macmillian, New York (1964), pp 195-203.

[3] F. Rohrlich, Classical Charged Particles, Addison Wesley, Reading, Mass, 1965. See particularly Ch. 2 and 6. See also pp 10-18, 128-129, 148, 157-160.

[4] D. K. Sen, Fields and/or Particles, Academic Press, New York, 1968, reviews dual and non-dualistic theories. Pages 41-43 review the theories of Mie and of Born and Infeld.

[5] J. D. Jackson, Classical Electrodynamics, 2nd Ed. John Wiley, New York (1975). p. 552.

[6] J. D. Logan, Invariant Variational Principles, Academic Press, New York, 1977.

[7] O. Consa, the General Science Journal, Spain, June, 2014 oliver.consa@gmail.com.

[8] S. Ghosh, A. Choudhury, and J. K. Sarma, Apeiron, Vol. 19, No. 3, July 2012, p. 247.

[9] M. H. MacGregor, the Enigmatic Electron, Kluwer Academic Publishers, London, 1992, ISBN 07923-1982-6. pp. 100-101.

[10] K. Nalty, "The Parson Ring Model for the Electron", Unpublished, July 23, 2013. kurtnalty.com/The_Parson_Ring.pdf

[11] A. L. Parson, "A Magneton Theory of the Structure of the Atom", Smithsonian Miscellaneous Collection, Pub 2371, 80pp (Nov 1915) \{Reprinted Pub 2419, V65, N11 (1916)\}.
[12] V. Simulik, What is the Electron? C. Roy Keys, Inc, Montreal, Quebec, 2005, ISBN 9-9732911-2-5, is a review of several models of the electron.

[13] A. A. Babin, and A. A. Figotin, Neoclassical Theory of Electromagnetic Interactions, Springer, Aug 4, 2016, ISBN97814471-7282-6.

[14] D. Blackmore, A. K. Prykarpatski, N. N. Bogolubov (Jr.), J. J. Slawianowski Mathematical foundations of the classical Maxwell-Lorentz electrodynamic models in the canonical Lagrangian and Hamiltonian formalisms, Universal Journal of Physics and Application Vol. 1 No. 2, 160-178, 2013.

[15] N. N. Bogolubov Jr., A. K. Prykarpatski , D. Blackmore, Maxwell-Lorentz Electrodynamics Revisited via the Lagrangian Formalism and Feynman Proper Time, Paradigm Mathematics 2015, Vol 3, No. 2, 190-257, 2015

[16] V.M. Simulik and I.Yu. Krivsky, "Slightly generalized Maxwell classical electrodynamics can be applied to inneratomic phenomena", Annales de la Fondation Louis de Broglie, Volume 27 no 2, 2002, pp. 303-328, use modified Maxwell's equations to describe hydrogen atomic states as well as "quantum mechanics can do".

[17] V.M. Simulik and I.Yu. Krivsky, "Relationship between the Maxwell and Dirac Equations: Symmetries, Quantization, Models of Atom”, Rep. Math. Phys., Vol. 50, N 3, (2002) pp. 315-328.

[18] V.M. Simulik and I.Yu. Krivsky, Classical Electrodynamical Aspect of the Dirac Equation, "Electromagnetic Phenomena", Vol. 3, No. 1(9), (2003) pp 103-114.

[19] J. S. de Wet, On the quantization of field theories derived from higher order Lagrangians, Proc. Camb. Phil. Soc. Vol 44. pp. 546-559, 1948 